\title{
RESISTANCE TO PERSUASION CONFERRED BY ACTIVE AND PASSIVE PRIOR REFUTATION OF THE SAME AND ALTERNATIVE COUNTERARGUMENTS ${ }^{1}$
}

\author{
WILLIAM J. MCGUIRE \\ Department of Social Psychology, Columbia University
}

$\mathrm{T}$ HE present study is one of a series comparing the relative effectiveness of various pretreatments in making initially unquestioned beliefs resistant to change when subsequently the person is forced to expose himself to strong counterarguments against the belief. The theoretical point of departure of the present and the earlier studies is the postulate of "selective exposure." Initially strong appearing beliefs, it is assumed, are actually quite vulnerable under forced exposure to strong counterarguments, because such beliefs tend previously to have been overprotected. By selective avoidance of counterarguments in the past, the person has kept his beliefs extreme, but has also left himself unpracticed in their defense and unable to deal with strong counterarguments when exposure to them is forced.

The postulate of "selective exposure" is no novelty in communication research. Since Klapper (1949) called it "the most basic process thus far established by research on the effects of mass media," it has stimulated much research, designed either to account for it in a theoretical system (Festinger, 1957) or to use it predictively (Janis, 1957). Its general relevance to the present problem is to suggest that beliefs can be "inoculated" against persuasion in subsequent situations involving forced exposure to strong counterarguments by pre-exposing the person to the counterarguments in a weakened form that stimulates-without overcoming-his defenses (Lumsdaine \& Janis, 1953).

The hypotheses tested in the study reported here were suggested by interpretations of the outcomes of two previous studies. One of them (Papageorgis \& McGuire, 1961) demonstrated that prior exposure to refuted counterarguments tends to make a belief more resistant to subsequently presented strong forms, not

\footnotetext{
1 This study was supported, in part, by a grant from the Office of Social Science, National Science Foundation. A partial version of this paper was presented at the 1960 APA meetings in Chicago (McGuire, 1960).
}

only of the very counterarguments refuted, but also of novel counterarguments against the belief. Such generalized immunization could derive from either of two mechanisms. Pre-exposure might shock the person into realizing that the "truisms" he has always accepted are indeed vulnerable, thus provoking him to develop a defense of his belief, with the result that he is more resistant to the strong counterarguments when they come. Alternatively the refutations involved in the pre-exposure might make all subsequently presented counterarguments against the belief appear less impressive. The other relevant study (McGuire \& Papageorgis, 1961) showed that when the subsequent attack is by the same counterarguments as were previously refuted, more resistance is conferred when the subject passively attends to the refutation than when he himself actively participates in it. That study corroborated the prediction from "selective exposure" that the person is so little able to defend these previously unquestioned truisms that the prior defensive session is not used effectively when the subject is called on to help refute the counterarguments. Hence, the particular counterarguments presented are more thoroughly refuted and subsequently better resisted in the passive defense situation than in the active.

The three main hypotheses tested in the present study all deal with interaction effects between type of prior refutation defense and whether the subsequent attack involves strong forms of the same counterarguments as were refuted or of novel counterarguments.

The first hypothesis would limit the previous finding (McGuire \& Papageorgis, 1961)that passive exposure to the refutations is more effective than active in producing resistance to persuasion-to situations in which the subsequently presented strong counterarguments are the very ones previously refuted. When, on the contrary, the subsequent exposure is to novel counterarguments against the belief, the present hypothesis holds that 
active defense is relatively more effective (and may even be absolutely more effective) in producing resistance. Of the two hypothesized mechanisms for the immunizing efficacy of the refutation defense-shocking the subject into developing support for his belief by acquainting him with its vulnerability and making subsequent counterarguments seem less impressive by refuting the earlier ones-the first is better provided by the active defense (wherein his inadequacy makes him feel more vulnerable) and the latter, by the passive defense (in which the counterarguments get more thoroughly refuted). Since the first mechanism is likely to be more important in providing resistance to strong forms of novel counterarguments and the second mechanism, to strong forms of the same counterarguments, the interaction prediction follows.

A second hypothesis deals with combinations of defenses: lengthening the prior exposure session to allow both active and passive defense should produce greater resistance increment over the single prior defense when the subsequent strong exposure is to the same counterarguments as had been refuted. Less difference in resistance between the single and double defense is predicted when the subsequent strong exposure is to novel counterarguments. The basis of this hypothesis is similar to that of the first one. The resistance to the specifically refuted counterarguments increases with the thoroughness of the prior refutation; the resistance to novel counterarguments depends more on the shock value of the pre-exposure, which is more likely to lessen than increase with the thoroughness of prior refutation of the counterarguments.

The third hypothesis deals with an order effect within the double defense condition: the passive-active sequence of prior refutation should be more effective than active-passive if the subsequent attack involves strong forms of the specifically refuted counterarguments; while the active-passive order should be the more effective against novel counterarguments. Similar reasoning again underlies this hypothesis. The active defense is likely to be more effectively carried out if it is preceded by the passive since in this case the subject does not have to depend on his own meager repertory of defenses when he is called upon to refute actively the counterarguments, but has at his disposal the refutations to which he has just passively attended. Hence, the counterargument to which he is pre-exposed should be more effectively refuted with the passive-active order, and he should show more resistance to these specific counterarguments.

The active-passive order, on the other hand, is predicted to be a more effective preparation for subsequent exposure to strong forms of novel counterarguments. When the active defense comes first, the subject has already had the dismaying experience of being confronted with novel counterarguments which he is hard pressed to refute, but he has then seen in the passive defense how effectively these formidable-seeming counterarguments can be refuted by an expert. After this reassuring experience, the subject is likely to be less dismayed by the further novel counterarguments used in the attack. $\mathrm{He}$ should tend to recall that the earlier counterarguments also appeared formidable when he was first called upon to refute them actively, and yet were shown to be quite refutable in the subsequent passive defense.

\section{METHOD}

\section{General Procedure}

All subject's took part in two one-hour experimental sessions. The first was devoted to the active and passive belief defenses; the second, to presenting the strong counterarguments against the belief. The persuasive aspects of the experiment were disguised by representing the study as an attempt to obtain norms for analytic thinking ability in high level personnel, a representation given credence by some of the tasks the subjects were called upon to perform.

First session. All the defenses to which this session was devoted consisted of a mention of two counterarguments against one of the four experimental beliefs, together with a refutation of these counterarguments. The refutations took one of two forms: either the subject was asked to read a mimeographed message (about 600 words long) that mentioned two counterarguments and refuted them in detail (passive defense); or the subject was given a sheet listing the two counterarguments and told (without any specific guidance) to show how each of these counterarguments could be refuted (active defense). The subject received passive only, or active only, or active-then-passive or passivethen-active defenses on one or another of the four experimental issues. In the passive defense condition, the subject was given 5 minutes to read the message and to select and underline the key clause in each paragraph (this task being introduced to bolster the 
"analytic ability" pretext for the experiment and to insure attention to the material). In the active defense condition the subject was given 10 minutes to refute the two counterarguments and was told that what he wrote need not reflect his own opinion and could be based on any relevant material he found in earlier parts of the test.

Second session. Two days later, the subject took part in the second session which was devoted to messages containing strong counterarguments against his belief. These messages were similar in appearance to the refutational messages used in the passive defenses conditions, each being about 600 words long and mentioning two counterarguments against the belief. However, while the defensive messages had been devoted mainly to refuting the counterarguments mentioned, these second session messages were devoted primarily to showing how the counterarguments were valid. Here again the subject was given 5 minutes to read and underline the crucial phrases of the message on each issue. In half the cases, the two counterarguments in these strong-attack messages were the same two as the subject had previously seen refuted; in the other half of the cases, the two counterarguments were novel.

After completing this "analytic reading and underlining" test, the subject was given the opinion questionnaire and asked to indicate his personal beliefs on the issues regardless of what materials may have been presented to him in the test. This questionnaire was justified to the subjects as needed to determine if the testee's attitude towards the content of the passages he had read affected the verbal skills in which we were supposedly interested.

Afterwards the subject filled out a questionnaire designed to test the effectiveness of the manipulations and experimental conditions and was given a detailed explanation of the nature of the experiments and the deceits used. Particular effort was made to assure the subject that all material presented in the messages was designed solely to persuade him in one direction or the other and that no special credence should be given to any of the arguments simply because it appeared in these messages.

\section{Materials}

Issues. Four health issues were selected on the basis of earlier studies which indicated that beliefs on items were homogeneously extreme in the college population. Three of the issues were used in a previous study (McGuire \& Papageorgis, 1961): "Everyone should get a chest $X$ ray each year to detect any possible TB symptoms.at an early stage"; "The effects of penicillin have been, almost without exception, of great benefit to mankind"; "Everyone should brush his teeth after every meal if at all possible." A fourth, used for the first time in the present study, was: "Everyone should see his doctor at least once a year for a routine medical check-up."

Opinion questionnaires. Opinions on these issues were measured by having the subject indicate on a 15-point graphic scale the extent of his agreement with statements bearing on one of the issues. There were 17 statements in all, 4 on each issue, the seventeenth being a repetition of an earlier item (to serve as a reliability check). The opinion scores to be reported are the mean number of points the subject obtained on the four items dealing with the issue in question.

Defensive messages. ${ }^{2}$ Each of the 600 -word passages used in the passive defense consisted of three paragraphs. The first stated the belief and mentioned two counterarguments against it, together with the remark that each was refutable. The second and third paragraphs were devoted to a detailed factual refutation of each counterargument. There were two such messages on each issue (hence, eight in all) in order to counterbalance material and implement the design of having half the subjects receive, in the second session, strong forms of the previously refuted counterarguments, and the other half, novel counterarguments. These messages were given appropriate titles ("Some Misguided Attacks on Penicillin," etc.) but not specific source attribution. They were mimeographed, single spaced, on a letter-sized sheet.

In the active defense condition the refutational material consisted of a sheet of paper with the same title and a brief statement of two counterarguments against the belief, together with instructions to write refutations of these counterarguments (not necessarily expressing one's own view and using any material one wished from previous parts of the test). Here again there were two different forms for each issue, each presenting an alternative pair of counterarguments to be refuted.

In the double defense conditions, the subject always received the same pair of counterarguments for refutation in the active as in the passive defense.

Strong counterargument messages. These messages, attacking the beliefs, were similar in length and format to the essays in the passive defense condition but differed radically in content. The first paragraph suggested that informed opinion has recently begun to question the "truism" and mentioned two counterarguments against it. The next two paragraphs attacked the belief by describing detailed factual evidence bolstering the counterarguments. Each message was suitably titled ("Some Drawbacks Involved in the Use of Penicillin," etc.). There were two messages attacking the belief on each issue, the two developing alternate pairs of counterarguments.

\section{Design and Subjects}

A total of 168 subjects took part in both sessions. In order to obtain data for all the comparisons involved in the hypotheses and also comparable data on some needed controls, a complex design was employed. There were four types of defense (active, passive, active-

${ }^{2}$ All 24 of the defensive and attacking messages used in this study have been deposited with the American Documentation Institute. Order Document No. 6866 from ADI Auxiliary Publications Project, Photoduplication Service, Library of Congress; Washington $25, \mathrm{D}$. C., remitting in advance $\$ 2.00$ for microfilm or $\$ 3.75$ for photocopies. Make checks payable to: Chief, Photoduplication Service, Library of Congress. 
passive, and passive-active), which could then be followed by no attack, attack with the same counterarguments, or attack with novel counterarguments, making for 12 conditions in all. In addition, there were two control conditions: an attack only condition, without prior defense, to ascertain the effect of the strong counterarguments when the beliefs had not been immunized; and a condition involving neither attack nor defense, to ascertain the "initial" belief levels. Each subject served in 4 of these 14 conditions, a different issue being used in each of his 4 conditions. The combinations of conditions given to any one subject were limited only by the requirements that all subjects be given tasks taking the same amount of time (one hour) in each session. The number of subjects serving in each condition is shown in Table 1.

Since the design called for four issues and two sets of material on each issue, counterbalancing the material required eight subgroups in the 12 attack-and-defense conditions, and in the thirteenth (attack only) condition; while in the fourteenth condition (neither-attacknor-defense) four different subgroups were required, one on each issue.

The significance levels reported in the Results section are based on $F$ tests in which the "error" variance is the residual variance in the conditions being compared with the treatment and (where appropriate) individual differences variance removed.

The subjects were 168 students enrolled in the introductory psychology courses at the University of Illinois. Almost all were either freshmen or sophomores and about half were males and half, females.

\section{RESULTS}

\section{General Effects}

As can be seen in the top row of means in Table 1, the four defensive conditions did not differ appreciably among themselves in regard to their direct strengthening effects on the beliefs, prior to exposure to counterarguments. Even the largest between-means differences, 0.80 points (between the active-passive and the passive-active conditions), is significant only at the .25 level.

Furthermore, the mean belief level after these defense only treatments does not differ sizably from those in the neither-defense-norattack control conditions. The overall mean of 12.99 in the four defense only conditions is only slightly superior $(F<1)$ to the 12.78 mean in the control condition. This lack of direct strengthening effect from the prior defenses is particularly interesting in view of the hidden reserves of resistance against subsequent strong counterarguments which (as will be discussed below) were conferred on the beliefs.
TABLE 1

Belief Strength (on a 15-Point Scale) in the VarIOUS CONDITIONS BEFORE AND AFTER ATTACKS BY THE SaMe OR DifFerent Counterarguments IroM Those to Which the Subject Had Been Pre-exposed during the Defense

(Number in parentheses is the number of cases for the cell)

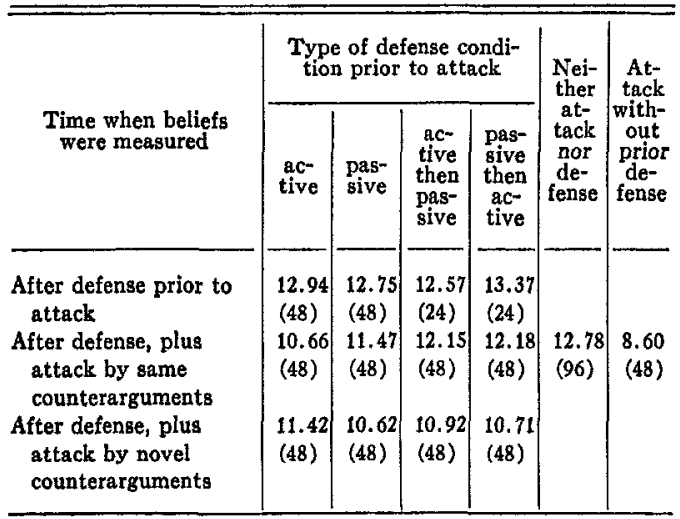

The beliefs were considerably weakened (to a mean of 8.60 ) by the strong counterarguments when these had not been preceded by any defense. This drop of 4.18 points from the control level is significant at the .001 level. The impact of these strong counterarguments was considerably lessened when they were preceded by a defensive treatment. The overall mean belief in the four defensive conditions after the defenses and strong attacks was 11.27. This does represent a 1.51 point drop from the control level $(p<.001)$, but it leaves the beliefs well above the 8.60 point level to which they were reduced when the strong attack had not been preceded by a defense.

\section{Comparative Immunizing Effectiveness of the Defenses}

Single defense conditions. The first hypothesis dealt with an interaction effect in the single defense condition: that while passive preexposure to refuted counterarguments tends to be superior to active in conferring immunity to subsequent strong forms of the same counterarguments, the active tends to be superior (or at least less inferior) to the passive in conferring resistance against novel counterarguments. As can be seen by comparing the four cells in the lower-left quadrant 
of Table 1 , this prediction was substantially confirmed. The interaction effect is in the hypothesized direction-passive defense superior against the same counterarguments, and active, against novel-and significant on the .02 level.

The nature of this interaction effect can be seen more analytically if we examine the comparative effectiveness of active and passive defense against the same and novel counterarguments, separately. With respect to immunization against strong forms of the same counterarguments, we find the final belief levels in the passive and active conditions to be 11.47 and 10.66, respectively. The difference between these subgroups is significant only at the .08 level, but corroborates the more significant result of the McGuire and Papageorgis (1961) study. Against novel counterarguments, we find the final belief levels in the passive and active defense condition to be 10.62 and 11.42 , respectively. Since this difference is significant only at the .13 level, it remains to be shown definitively that the active defense is actually superior to the passive with respect to conferred resistance against novel counterarguments. The significant interaction does, however, demonstrate that any superiority of passive over active defense is less pronounced against novel than against the same counterarguments.

In this study, as in that by Papageorgis and McGuire (1961), we find that the pre-exposure (either actively or passively) to refuted counterarguments produces considerable generalized immunity. In neither active nor passive condition is there a significant difference between the resistance to same and novel counterarguments, and in the two conditions combined the mean beliefs after the same and novel strong counterarguments (11.06 and 11.02 , respectively) is only trivially different. This finding is encouraging in regard to the possibility of making given beliefs immune to persuasion even when we cannot foretell which counterarguments will eventually be offered against them.

Single vs. double defenses. The second hypothesis dealt with an interaction between single vs. double defense and subsequent exposure to strong forms of the same vs. novel counterarguments. An inspection of the four means shows that both marginals, as well as the predicted interaction, attained appreciable levels of significance. The same vs. novel counterarguments marginal was significant at the .01 level: over the four defensive conditions, subsequent exposure to strong forms of the same counterarguments reduced the beliefs only to 11.61 points while novel counterarguments reduced them to 10.92 points.

The single vs. double-defense marginal shows the least differential (significant only at the .10 level): after the single defense the strong counterarguments reduced the beliefs to 11.04 points and after the double defense, to 11.49 points.

The interaction was in the predicted direction: the single defense was almost as effective against strong forms of novel counterarguments as against strong forms of the same counterarguments (the postattack means being 11.06 and 11.02 , respectively), while the double defense was much more effective against the same than against novel counterarguments (the postattack means being 12.17 and 10.82, respectively). This interaction is significant at the .01 level. To state this interaction effect in another way, the slight marginal superiority of the double over the single defense just mentioned $(p=.10)$ was due entirely to the subconditions in which the subjects were subsequently exposed to the strong forms of the same counterarguments as had been refuted in the defensive session. In this "same" condition the superiority of double over single defense was significant at the .001 level. The implication is clear that it is worth belaboring the counterarguments to the extent of a double (active and passive) refutation if and only if the subsequent attack is going to involve the very counterarguments refuted.

That the single defense is as good as-in fact, trivially $(p=.60)$ better than-the double in conferring resistance to novel counterarguments fits in well with the underlying postulate that the efficacy of the prior refutational defense in producing resistance to novel counterarguments derives mainly from provocative impact of pre-exposing the belief to counterarguments, which brings home to the subject that the truism is indeed attackable and stimulates him to bolster his belief. Elaborate double refutation of the 
counterarguments adds little since the attack involves novel counterarguments (and may subtract from the defense stimulating impact by refuting too thoroughly the counterarguments to which the subject is pre-exposed during the defense). Thus this interaction effect between the single vs. double defense and the same vs. novel counterargument variables lends itself to the same theoretical interpretation as did the significant interaction between the passive vs. active single defense and the same vs. novel counterargument variables.

The double defense conditions. The third hypothesis dealt with an interaction effect predicted in the double defense condition: that the active-then-passive sequence of defenses would prove more resistance producing against strong novel counterarguments, while the passive-then-active sequence of defenses would be more effective against strong counterarguments similar to those refuted. As can be seen by inspecting the four relevant means in Table 1 (lower center portion), the interaction effect between the order of defenses and the same vs. novel counterarguments variables is in the predicted direction but trivial in magnitude. Neither is there any differential effect in the sequence margin per se: the strong counterarguments reduced the beliefs to 11.54 points after the active-then-passive sequence, and to 11.45 points after the passivethen-active sequence of defenses. (The $F$ of this difference is less than 1.) The only significant difference in the double defense condition is in the same vs. novel marginal: after the double defenses, the beliefs were weakened only to 12.17 points by strong counterarguments that were the same as had been refuted, but to 10.82 points by novel counterarguments. This difference is significant on the .001 level. As mentioned in the previous section, this sizable same-vs.-novel effect in the double defense conditions contrasts with the lack of such effect in the single defense, which contrast tends to confirm the initial postulate of the study.

While the preceding discussion demonstrates that the eight defensive conditions differed considerably among themselves in their immunizing effectiveness, it should be noted also that all conferred on the belief a significant resistance to the subsequent strong attack. Even the least effectively immunizing defense (single passive defense, with subsequent attack by novel counterarguments) left the beliefs still appreciably stronger after the attack (at a mean of 10.62 points) than they were left in the attack only condition, where the resulting mean was only 8.60 , the difference being significant at the .001 level.

All the beliefs being defended and attacked in this study were of a highly homogeneous type: all were cultural truisms and all touched upon a highly involving and anxiety arousing topic, physical well-being. Perhaps this deliberate homogeneity is responsible for the obtained generality of effects across issues, in the sense that none of the interaction effects between issues and type of immunization reached conventional levels of significance. (However, issues as a main effect did contribute significantly $(p<.05)$ to the variance; that is, the overall belief levels after both combined defenses and attacks did vary significantly among the four issues.) Generalization of these results to other types of issues would be unwarranted without further research.

There are, in fact, theoretical reasons for hesitating to generalize the present findings to other types of beliefs. The "selective exposure" postulate used to derive the present prediction would yield quite different predictions with other types of beliefs. Had the beliefs been controversial rather than truisms, the subjects would have been more practiced in defending them and, hence, would have participated more effectively in the activedefense condition and would have been less in need of a threatening, defense stimulating pre-exposure. Had the issues been less involving than these health ones, there would have been more to gain, in regard to motivating the subject to pay adequate attention to the material, from requiring his active participation in the defense. Hence, with different types of issue we would expect resulting differences not only in the size of the obtained effects, but even in their directions.

\section{SUMMARY}

The postulate that cultural truisms tend to be overprotected as a result of the tendency toward "selective exposure," together with 
the results of earlier studies, led to three predictions regarding the amount of resistance to subsequent strong counterarguments that would be conferred by pre-exposure to refuted counterarguments under conditions of active, passive, and combined refutation. The hypotheses dealt with interaction effects between amount of activity during the pre-exposure and whether the strong counterarguments used in the subsequent attack were the same as or different from those to which the subject had been pre-exposed.

Each of the 168 college student subjects served in four conditions, each on a different issue. The complex design furnished data on the relative effectiveness of the four defensive conditions (active, passive, passive-active, and active-passive refutations of counterarguments) in regard to direct strengthening effect, and immunization against the same, and against novel counterarguments presented in strong form 2 days later.

Besides corroborating various results of previous studies, the present results confirmed two of the three interaction hypotheses.

1. In the single defense condition, more immunity was conferred against the same counterarguments by passive than by active defense, while against novel counterarguments the active defense was the more effective $(p=.02)$.

2. In the double defense condition the predicted interaction effect involving the sequence of the active and passive defenses was not found.

3. As predicted, the immunizing superiority of the double over the single defense was found only when the subsequent attack involved the same counterarguments; against novel counterarguments, the single defense was as effective as the double (interaction significant at the .01 level).

\section{REFERENCES}

Festinger, L. $A$ theory of cognitive dissonance. Evanston: Row, Peterson, 1957.

JANIS, I. L. Motivational effects of different sequential arrangements of conflicting arguments. In C. I. Hovland et al., The order of presentation in persuasion. New Haven: Yale Univer. Press, 1957. Pp. 170-186.

KLAPPER, J Effects of the mass media. Columbia University, Bureau of Applied Social Research, 1949. (Mimeo)

LumSDatNe, A. A., \& JaNis, I. L. Resistance to "counter propaganda" produced by one-sided and two-sided "propaganda" presentations. Publ. opin. Quart., 1953, 17, 311-318.

McGoIrs, W. J. The relative effectiveness of active and passive prior defense in immunizing beliefs against persuasion. Amer. Psychologist, 1960, 15, 433. (Abstract)

McGuire, W. J., \& Papageorgis, D. The relative efficacy of various types of prior belief defense in producing immunity against persuasion. J. abnorm. soc. Psychol., 1961, 62, 327-337.

Papageorgis, D., \& McGutre, W. J. The generality of immunity to persuasion produced by preexposure to weakened counterarguments. $J$. abnorm. soc. Psychol., 1961, 62, 475-481.

(Received September 30, 1960) 